\title{
The structure of the Plasmodium falciparum 20S proteasome in complex with the PA28 activator.
}

Riley D. Metcalfe ${ }^{1}$, Stanley C. Xie ${ }^{1}$, Eric Hanssen ${ }^{1,2}$, Tuo Yang ${ }^{1}$, David L. Gillett ${ }^{1}$, Andrew Leis ${ }^{2}$, Craig J. Morton ${ }^{1}$, Michael J. Kuiper ${ }^{3}$, Michael W. Parker ${ }^{1,4}$, Natalie J. Spillman ${ }^{1}$, Wilson Wong ${ }^{5}$, Christopher Tsu $^{6}$, Lawrence R. Dick ${ }^{6}$, Michael D.W. Griffin ${ }^{1}$ and Leann Tilley ${ }^{1}$

${ }^{1}$ Department of Biochemistry and Molecular Biology and ${ }^{2}$ Advanced Microscopy Facility, Bio21 Molecular Science and Biotechnology Institute, The University of Melbourne, Melbourne, VIC 3010, Australia, ${ }^{3}$ Data61 CSIRO, Docklands VIC 8012 Australia, ${ }^{4}$ Australian Cancer Research Foundation Rational Drug Discovery Centre, St Vincent's Institute of Medical Research, Fitzroy, Victoria 3065, Australia, ${ }^{5}$ Infection and Immunity Division, The Walter and Eliza Hall Institute, Parkville, VIC 3052, Australia, ${ }^{6}$ Oncology Clinical R\&D, Takeda Pharmaceuticals International Co., Cambridge, Massachusetts 02139, USA

Email Contact: rileydm@student.unimelb.edu.au

The proteasome is a multi-subunit enzyme complex that is responsible for most of the non-lysosomal proteolysis in eukaryotic cells. The $20 \mathrm{~S}$ proteasome core is comprised of four heptameric rings, two rings of $\beta$-subunits, which contain the catalytic sites, sandwiched by two $\alpha$-rings, which control substrate access to the proteasome core. The malaria parasite, Plasmodium falciparum is highly reliant on its protein turnover machinery, thus the proteasome is a drug target in the treatment of malaria. The activity of the $20 \mathrm{~S}$ proteasome is regulated by protein complexes, such as the 19S complex, that bind to the $\alpha$-subunit rings. A less-well studied regulator is the PA28 (11S) activator, which stimulates proteasome activity independent of ubiquitin or ATP.

Here, we characterized PA28 from P. falciparum (PfPA28). We showed that genetic deletion of PfPA28 renders parasites more sensitive to anti-malarial drugs, consistent with a role for PA28 in responding to proteotoxic stress. We solved the crystal structure of PfPA28, revealing a bell-shaped structure with a highly charged central channel (Figure 1a). We purified $P f 20$ S from parasite cultures and structurally characterized the PfPA28-Pf20S complex using single-particle cryoEM. We solved the structure of $P f 20 \mathrm{~S}$ in complex with one and two PfPA28 caps to a resolution of $3.92 \AA$ and $3.82 \AA$ respectively (Figure 1b). These structures provide insight into the binding and activation mechanism of PfPA28 and provide further evidence that $11 \mathrm{~S}$ activators employ a distinct mechanism of activation compared to the 19S complex. Analysis of the cryo-EM data also showed that PfPA28 and Pf20S form a dynamic complex, with PfPA28 undergoing large rigid motions on $P f 20 \mathrm{~S}$. We propose lateral transfer of proteasome products through this interface as an alternative mechanism of substrate egress, avoiding the need for products to traverse the PfPA28 pore. This work adds to a growing body of knowledge on the structural basis of proteasome activation.

A

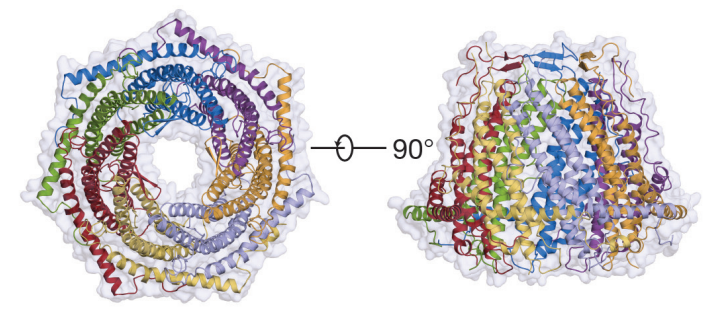

B

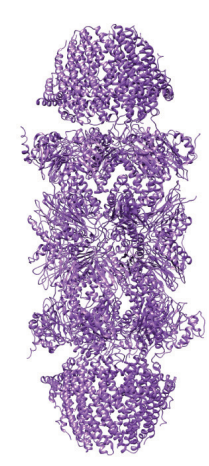

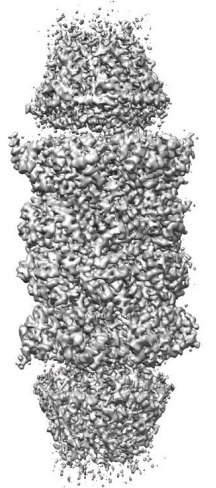

PA28

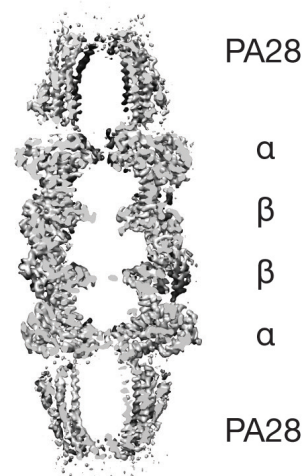

Figure 1: The structure of the $P f 20 \mathrm{~S} / P f \mathrm{PA} 28$ complex. A) shows the $3.1 \AA$ resolution crystal structure of PfPA28, showing a bell-shaped heptameric protein with $\sim 20 \AA$ diameter pore. B) shows the structure and cryo-EM density of the $3.8 \AA$ resolution double-PfPA28 capped Pf $20 \mathrm{~S}$ complex. A continuous channel is formed in the complex, allowing substrates to pass through the complex. 